\title{
CLASSIFICATION OF VIOLENT VIDEOS USING ENSEMBLE BOOSTING MACHINE LEARNING APPROACH WITH LOW LEVEL FEATURES
}

\author{
Snehil G Jaiswal \\ Research Scholar, Sant Gadge Baba Amravati University, Amravati \\ Officiating Registrar, G H Raisoni University, Amravati \\ Maharashtra, India \\ Mr.Snehil.Jaiswal@gmail.com \\ Dr. Sharad W Mohod \\ Professor, Department of Electronics and Telecommunication Engineering \\ Prof. Ram Meghe Institute of Technology \& Research \\ Maharashtra, India \\ sharadmohod@rediffmail.com
}

\begin{abstract}
With the fast development of monitoring video capturing gadgets to keep an eye on the human movement requires such system which perceive the suspicious, violent and dubious occasions without requiring no human intervention. Anomalous, abnormal and violent activity identification has become a prominent field of research in areas of human-computer interaction, computer vision, machine learning and digital image processing. This paper proposes a distinct technique of violent video classification, which depends upon fusion of low-level features extracted from specific frames of video since using the whole video as input may add both redundancy and noise in the learning process. We leverage low level features, and aggregate them by training a model using adaptive boosting an ensemble learning algorithm to achieve a task of Classification of Violent Videos as a binary-classification problem. The proposed technique is evaluated with three distinct datasets that are majorly used in the studies classification of violent videos. And it exhibits cutting edge classification capacity when given the task of differentiating violent and normal videos across wide variety of violent data including both crowded scenes and non-crowded scenes. The proposed work archives competitive results with an accuracy of $90.62 \%$ on hockey fight, $91.70 \%$ on movies, and $94.3 \%$ on crowd violence. The results indicates that in the view of human violence detection, our method is simple, unique and effective.
\end{abstract}

Keywords: Action recognition Violent behavior detection, intelligent video surveillance; computer vision Violent video Classification, Violence detection, Ensemble Boosting.

\section{Introduction}

More extent of the world's Internet traffic at present is occupied with video contents. Video contents majorly involving short video and live streaming is the latest trend of digital world. However, the larger proportion of inter video is crowded with some violent videos, which are surely harmful to modern civilized society in one or many ways.

Human action recognition is challenging research area where the focus is on modeling a human activity detection and recognition system. Violence detection and classification in video is the special case of action recognition, wherein detection of violent scene and violent content and in video is performed by using visual and auditory (if available) feature extraction algorithms and then by classifying it using suitable classifier.

The factors like intra-class varieties of human movement, the changing background, variation of scale and viewpoint makes the violence detection and classification a difficult task. Furthermore, detecting and monitoring the violence in time lay downs another hurdle in front of violence detection system. Thus, framework developed to detect the violent content in video shall be a fast, reliable, robust method and is consequently expected to work continuously in real time. Thus, development of violent video detection and classification algorithms 
based on Computer vision and machine learning techniques are highly demanded for intelligent surveillance and automatic video annotation and are of primary importance.

In this paper, our research contributions can be summarized as follows:

- Exploration of low-level spatiotemporal features of the video frames for violence detection.

- Coalescence of right combination of feature for a perfect feature vector.

- Efficient and accurate classification using machine learning meta-algorithm.

- Experimental analysis on real-world datasets which are commonly used.

The rest of the paper is organized in five main sections. Section II discusses the various methodologies reported in literature based on the literature review related to the violence detection. Section III presents the proposed approach of violent video classification. In Section IV, the evaluation of proposed methodology on benchmarking datasets are deliberated in detail and in the end, section V summarizes the paper with the conclusion

\section{Related Work}

Violence detection in video is a kind of action detection problem in computer vision area. Several different methods are proposed that uses different input parameters present in the video such as appearance, optical flow, time scale related parameters. The generalized violence detection problem can be formulated as three step problems involving first step as extraction of shots / frames, second step as extracting a useful information present (features) describing the scene and third step as to classification using suitable classifier [Ramzan et al., (2019)] [Jaiswal and Mohod, (2020)]. However, the steps may vary slightly according to method applied for detection. This section reviews the various aspects such as features and classifiers that are used for violence detection in video using computer vision system. In this section analysis of available research is presented.

Authors in [Zin and Kurohane, (2016)] has works on a novel approach for human interaction is shown in which silhouette contour features detect human activity. This has been achieved in three steps: the information of foreground object is extracted using an adaptive background model in first step and this foreground object is converted into the form of the silhouette. The features from detected object are extracted in second stage which is given as input for classification using suitable classification algorithm. The structure of human action recognition system is shown in fig. 1. [Zin and Kurohane, (2016)]

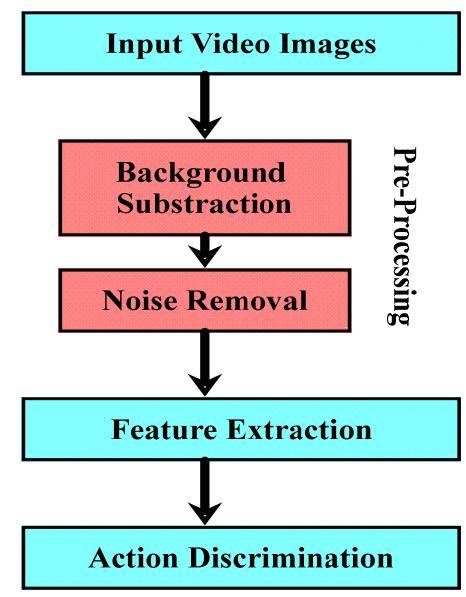

Fig 1: System Flowchart of Human Interaction [(Zin and Kurohane, 2016)]

A new modified and improved algorithm for violence detection from motion images is proposed in [(Zhang et al., (2017)]. First, they introduced an enhanced Weber local descriptor to extract more information from lowlevel images and then extend the spatial descriptor and add temporal component to represent motion image information. This second modification is based on sparse representation classification model which is used to control the reconstruction errors as well as classification error to minimize. The sparse model, a class-specific dictionary is provided that contains class labels of training samples. With the assistance of this dictionary, representation residual and representation coefficient become discriminative[(Zhang et al., (2017)]. 


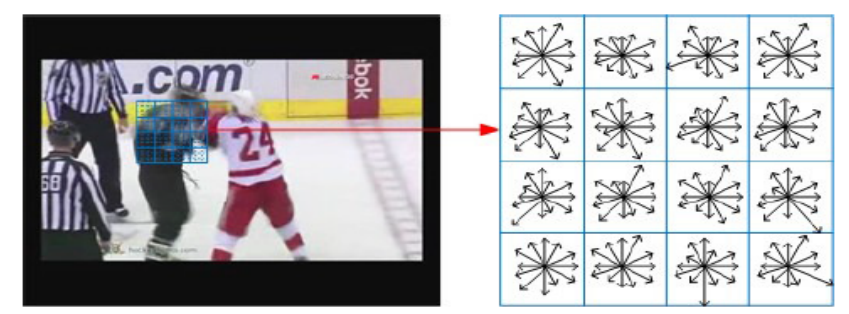

Fig. 2. Grid Aggression for Weber Local Descriptor [(Zhang et al., (2017)].

Introduction of a new technique to overcome the traditional methods like spatiotemporal description and bag of words method is proposed in [Xu et al., (2014)] with MoSIFT algorithm known as Motion SIFT which extract the low-level description of an input video. KDE (Kernel Density Estimation) method is use to remove the feature noise. This paper also endorses the sparse coding scheme to obtain highly discriminative video feature. Normalized probability density function by Kernel density estimation method is as shown in fig.3.[Xu et al., (2014)]

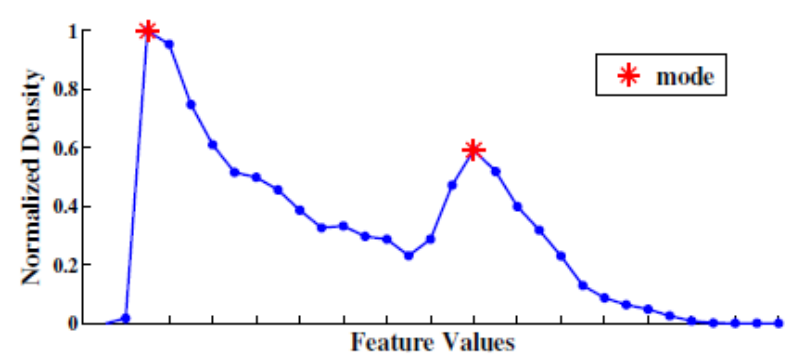

Fig. 3. Normalized Probability Density Function By Kernel Density Estimation Method [(Xu et al., 2014)]

Authors in [Wang and Kato, (2016)] came up with a concept to estimate the visual violence extent from the given video. Such technique can be used in several applications where one is required to restrict the access of violent videos to teenagers. They proposed an approach for violence rating featured with an innovative video descriptor called VAA (Violent Attribute Activation) vector, provides the high-level description on properties of visual violence; and a rating approach based on rank prediction for enforces the order constraints in the learning process.[Wang and Kato, (2016)]

The concern about the crime frequently occurred in the elevator cage is covered in [Tang et al., (2009)]. They proposed intelligent video analysis for crime detection in an elevator cage. By collecting the real-time video images, the necessary data is then processed and analyzed via K-Means clustering to get observation sequences, which are used to model a Hidden Markov Model (HMM) for regular activity. In the last step, the difference between the abnormalities and normal activity mode is taken, and by observing the sequence of normal active mode, the standard value is predetermined. [Tang et al., (2009)]

Development of a technique for the automatic analysis of videos to recognize the violence present in the input video using a neural network is achieved with the help of system in [Sudhakaran and Lanz, (2017)]. A convolutional neural network method is used to extract frames and then by using convolutional gates frames are aggregated using long-short-term memories. This convolutional method is capable of capturing the spatiotemporal feature for the analysis of local motion taking place in the video.[Sudhakaran and Lanz, (2017)]

Authors in [Souza and Pedrini, (2017)] worked on a novel method for violence detection using CENTRIST based features. Primarily the video is preprocessed so as to improve the over all quality of the object in the scene and to decrease the noise effects like lightning changes. Then, the CENsus Transform histogram (CENTRIST) is used to extract the set of features from the video frames. To reduce noisy information, the dimensionality of the frames is also tested. Finally, the video frames are classified as violent or non-violent. [(Souza and Pedrini, 2017)]

A real-time monitoring system which not only detects people but also monitors their behaviors in open and occult environments is described in [Serrano et al., (2018)]. Such a detection system can be applied to elevator cage abnormality detection.

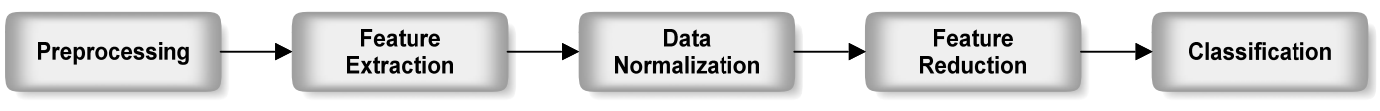

Fig.4. Main Stages of Proposed Work in [Souza and Pedrini, (2017)]

To prevent crime authors in [Souza and Pedrini, (2017)] introduced CCTV base systems beneficial in work environment and public places and transport, using signal processing and learning algorithms but the objects 
involve are different. In the proposed method one of the aims is to identify the critical conditions during official meetings and discussions using images and speech processing and ensure conditional video recording. However, using video and voice processing the target is to identify the critical movements, to alert the nearby surveillance station. The method can be useful to prevent interpersonal violence and crime against women. [Souza and Pedrini, (2017)]

Paper [Senst et al., (2017)] disclosed the Lagrangian theory based lagrangian direction field based on the spatiotemporal model. To describe non-linear dynamic systems lagrangian methods are used. Lagrangian fields are derived by analyzing different types of integral curves within the time-dependent flow field. An overview of different field line types is shown in fig. 5. Streamlines represent a point of zero velocity or noise. Path lines plots a single seed point to a new position at each point in time denoted as flow map. Streaklines are used to observed physical interactions in the numerical simulations. Timeline gives the progression of given seed structure over time and during integration in the flow field captures its deformation [Senst et al., (2017)].
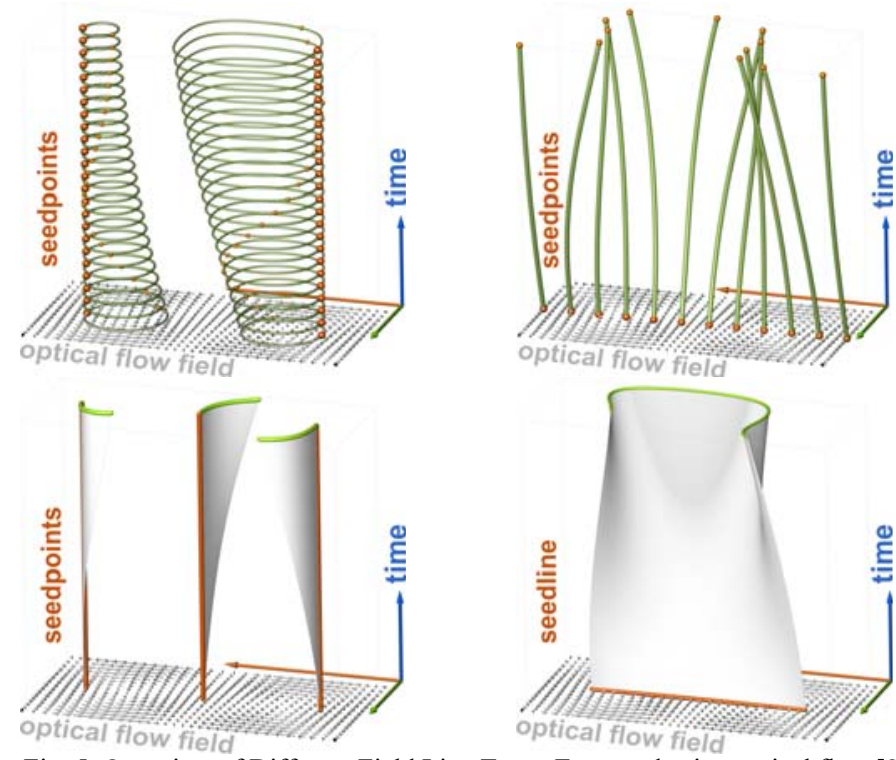

Fig. 5. Overview of Different Field Line Types Extracted using optical flow [Senst et al., (2015)]

System in [Senst et al., (2015)] introduced Lagrangian theory for the detection and classification of violent video and non-violent video. A local feature based on the SIFT algorithm based on Lagrangian motion models. The evaluation of an algorithm is based on a bag of word technique which shows some better results over state of the art on current violent detection datasets. [Senst et al., (2015)]

A method for violence detection in a video for crowd activity detection is proposed with system developed in [Keçeli and Kaya, (2017)]. They came up with a novel technique for violence detection as the transfer learning-based method with a guarantee of better result compared with existing detectors. First, using the Lucas Kanade method, the optical flow of the input videos is compute. Then, several 2D templates are generated with overlapping optical flow magnitudes. These templates are applied as an input to the convolutional neural network, and deep features of different layers are extracted. [Keçeli and Kaya, (2017)]

Vector machine and nearest neighbors' classifiers are trained for the prediction of violence scene. Flowchart for proposed method is shown in Fig.6. 


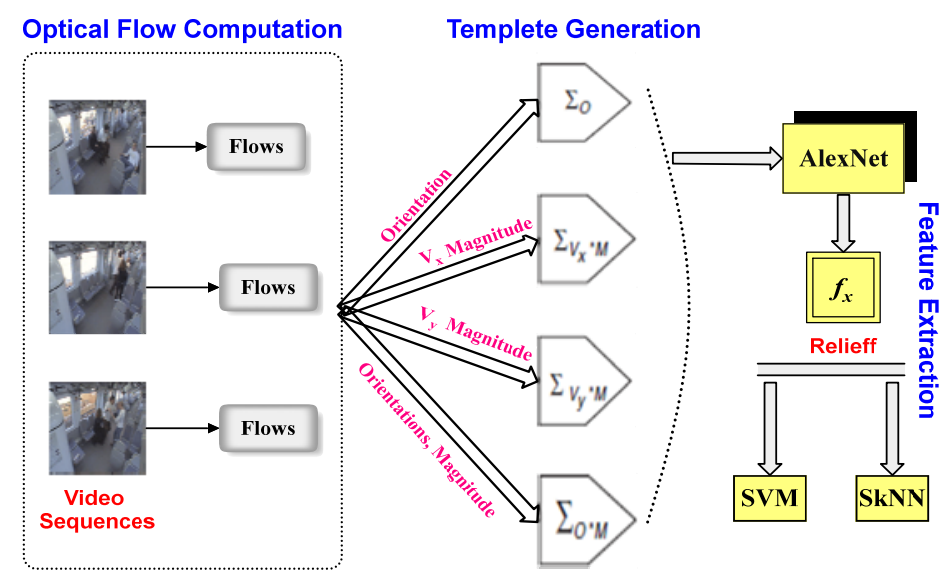

Fig.6. Proposed method Flowchart in [Keçeli and Kaya, (2017)]

Markus Horhan et al. came up with the concept that Real violence is an unwanted part of any video surveillance system and detection of abuse by the motion of hands is again an impossible task. This paper proposed the differentiation of violence and material art videos using two new feature transformations for jitter detection and local interest point detection with Gestalt laws.[Horhan et al., (2013)]

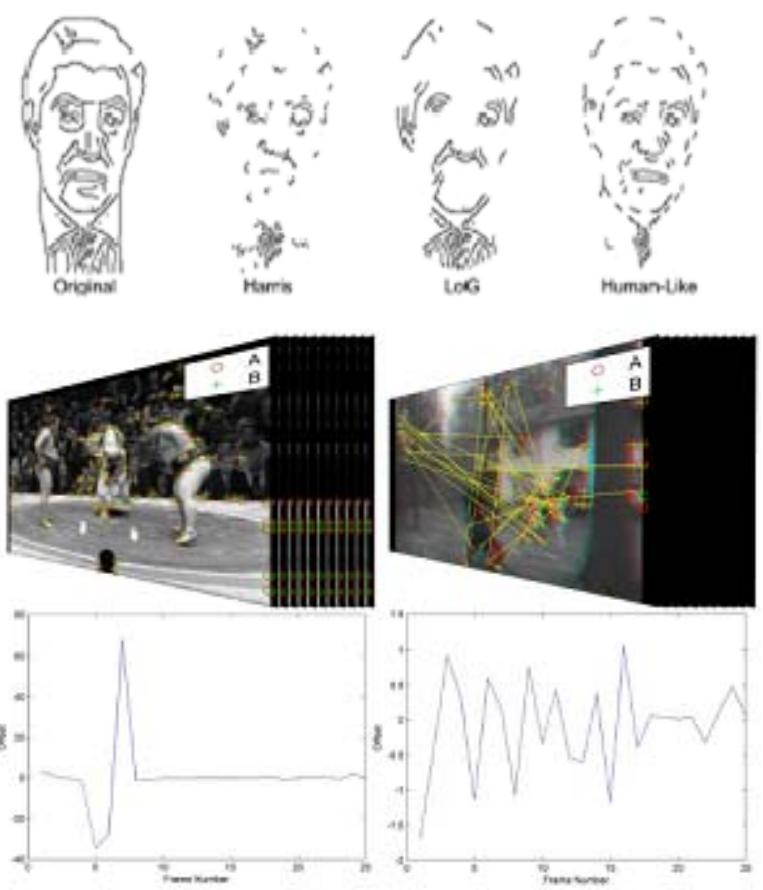

Fig. 7. Gestalt Interest Points [Horhan et al., (2013)]

Oscar et al. proposed a technique that measures the acceleration of overall motion by comparing the power spectrum of successive frames. Codebook-based on fisher vectors is used. These dynamic features are then quantized and gathered into fixed dimension histograms of the video. Classification is done by vector machines.

[Khokher et al., (2015)] presented a new method for violence detection using super descriptor tensor decomposition technique. In this technique, multi-modal local features are extracted from video including first and second order derivatives. In the first step, the extracted feature is encoded using the super descriptor method. Then to retain the spatiotemporal structure the encoded features are arranged as tensors. An output of the proposed method gives an accuracy of $60.2 \%$ and $67.8 \%$ on two subsets of the dataset.[Khokher et al., (2015)]

[Hassner et al., (2012)] Gives a novel technique in which from optical flow information, violence flow descriptor is constructed. First, optical flows between consecutive frames are computed, and binary indicators 
are obtained from these flows. This, a histogram is constructed which consist of a vector of frequencies of the quantized value.[Hassner et al., (2012)]

[Cristani et al., (2007)] proposed a novel violent shot detection method for both audio and video aspects like the motion of images and blood analysis. Later on, he focused on skin and blood detection in video sequences which require foreground segmentation or the information of skin color. This feature degrades its performance to a greater extent when the skin color was not discriminating enough.[Cristani et al., (2007)]

\section{Proposed System}

Motivated by various insights of various technologies in literature, we take advantage of low-level features and simple classifier to proposed a better approach for violent video classification. The generalized framework of proposed methodology is illustrated in Fig. 1. The operation of present invention will be described below with respect to Fig. 1.

For developing a generalized violence detection model, a due care has been taken regarding training and testing of model. Firstly, the balanced splitting of entire database is done into two categories as training dataset and validation dataset. The $70 \%$ of the total dataset available is reserved for training phase and rest $30 \%$ for the testing phase. This splitting is done in a randomize manner every time whenever the experimentation is performed. This balanced splitting ensures the unprejudiced evaluation of a model fit on the training dataset with tuning model hyperparameters.

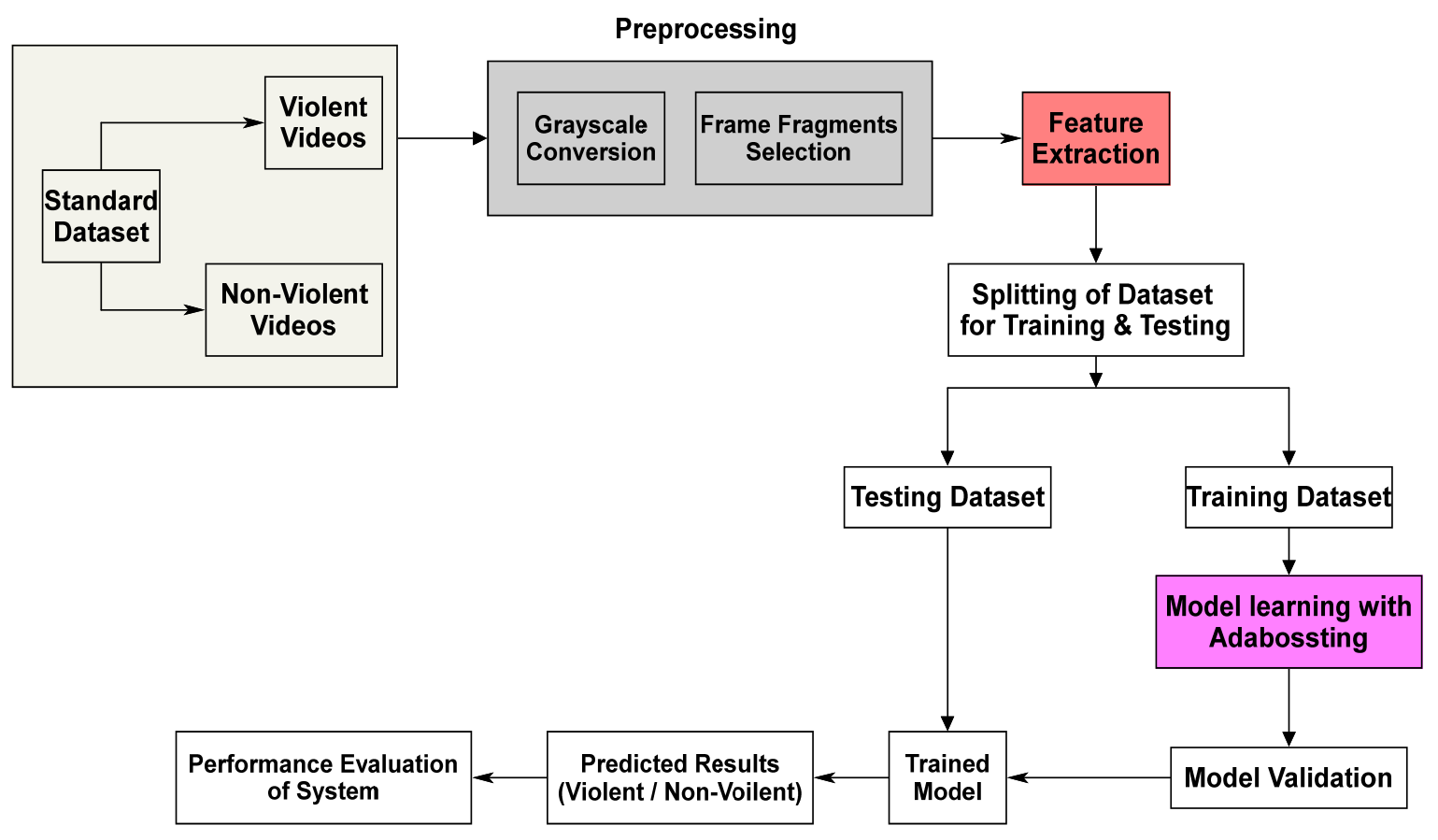

Fig 8: Proposed Violent Video Classification System

Firstly, the training dataset and testing dataset shall be passed through preprocessing unit, which comprises of Grayscale conversion unit and the Frame Fragment Extraction unit. Frame extraction can be defined as the process of taking out frame or set of frames that contains most of the valuable information in the context of feature extractions. By this process the prominent features of a video are preserved and most of the redundant frames are removed.

The extracted frames are the most accurate and compact summary of the video content. Preprocessing operations improves the overall quality of the raw data and makes it ready for extracting available information. In initial the training phase of the system, the preprocessed video data is then applied to feature extraction and fusion unit, wherein the motion features and temporal features (time domain features) i.e., Fuzzy Histogram of Optical Flow Orientations (FHOFO) and Multi-Resolution Local Binary Pattern (MRLBP) are extracted from video data. Feature extraction and fusion unit is equipped with not only facility to extract the feature but also to choosing a correction combination of features

Supervised learning algorithms are the class of machine learning, in which the model is developed by training the machines using well labelled training data, and then making use of this trained data, machines predict the output. A classifier unit adopting supervised machine learning algorithms is built on the basis of ensemble adaptive boosting approach. 
The journey of training dataset completes after passing through preprocessing unit, feature extraction and fusion unit and classifier unit to get a primary violence detection model. In validation phase, system evaluates the various performance evaluation parameters and accordingly adjust / tune the different model hyperparameters so as to give a trained violence detection model which can be used to classify any video.

When the input from testing dataset or video capturing device or any pre-stored video is applied to pretrained violence detection model, it will be classified as violent or non-violent with the assistance of trained violence detection model. If there is no violence detected in present sequence or present instant, the system will keep checking for next sequence in a continuous manner.

\subsection{Preprocessing}

The video data quality in case of video captured from devices like cctv may be low because of poor camera quality, surrounding noise, and limited transmission bandwidth \& storage capacity. In this manner, there are common preprocessing operations that must be performed on raw video sequence to prepare it for additional future extraction. The preprocessing unit improves the quality of video \& image; upgrades the digitized video \& image, so progressively exact data (features) can be taken out \& examine.[Jaiswal and Mohod, (2021)]

Preprocessing is required to reduce the training time requirement of model and subsequently increase the model inference speed.

\subsubsection{Grayscale conversion}

The conversion from true colour RGB image to grayscale image is achived using inbuilt function rgb2gray of matlab, which converts a RGB image into a image with pixel values ranging between 0-255 (8-bit unsigned integers). Fig. 1 (a) shows a sample input frame from dataset belonging to violent videos and its subsequent conversion to grayscale in (b).
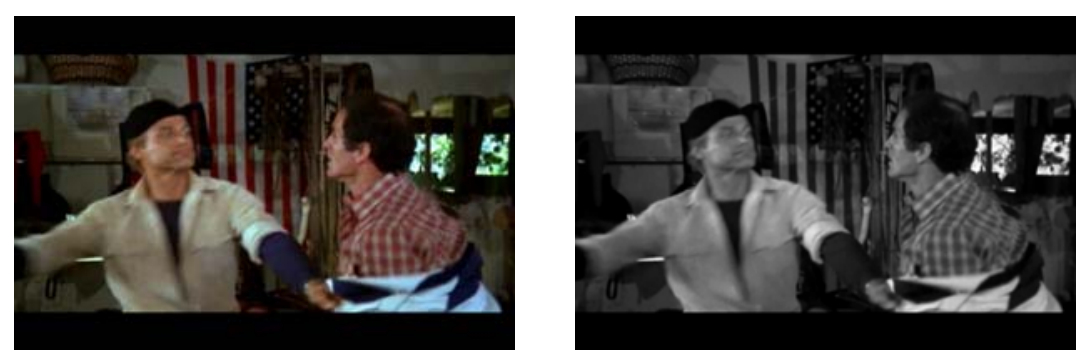

Fig 9: Grayscale Image conversion example. (a) is the original image form Peliculas dataset and (b) is the corresponding conversion of (a) to grayscale.

\subsubsection{Frame Fragment Selection}

Given a video clip, it is possible that violent action (which is our area of intertest) occurs only in specific part of the clip and prominently in some of the frames one can see the motion of person which is necessary to contribute violent or any other action. Majorly it is seen that there are no human actions without motions. For detecting frames involving major events of violent data, it uses Mean Square Error (MSE) estimation of two successive frames.

The working of frame fragment selection unit can be understood using following flowchart in Fig. 3. 


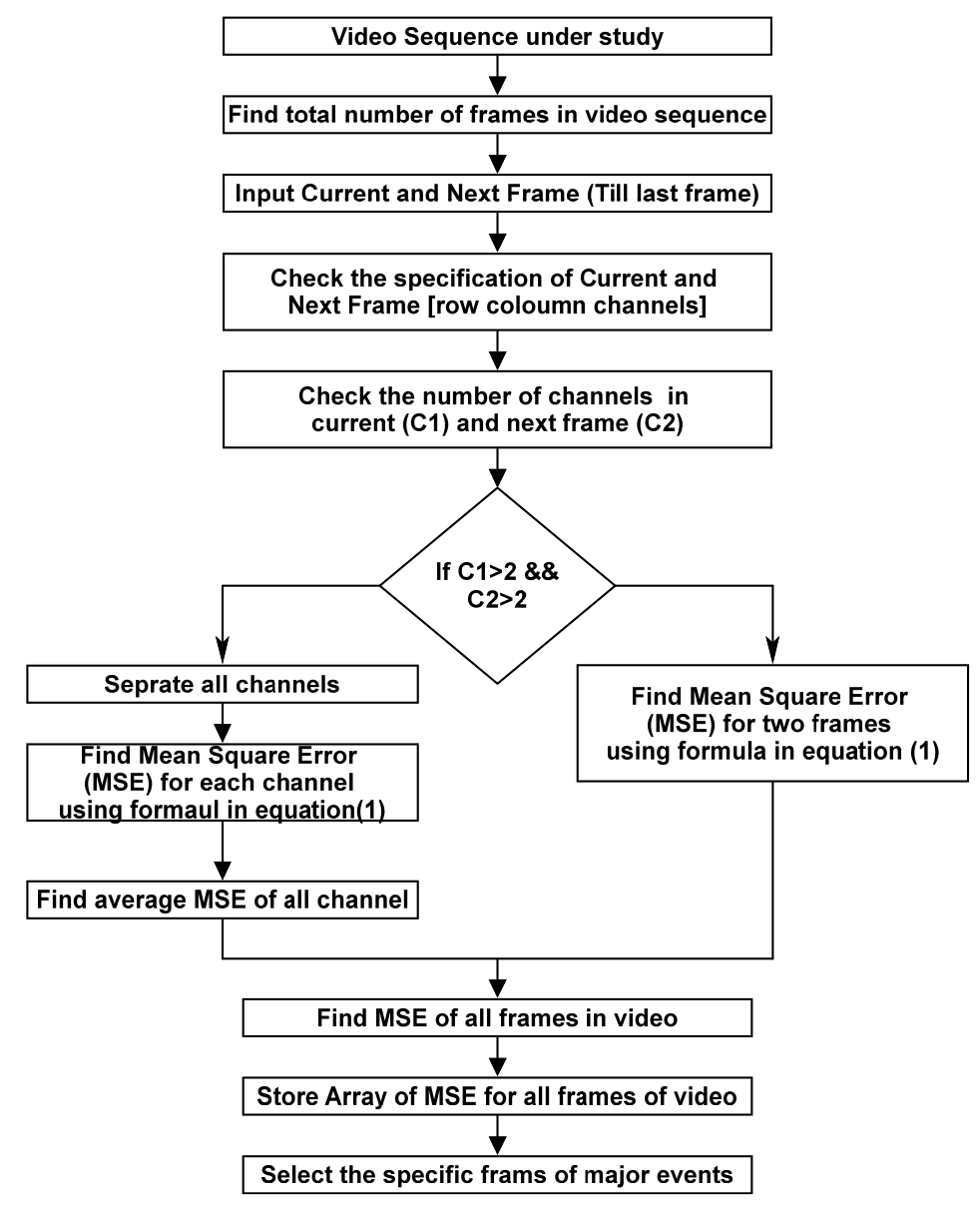

Fig 10: Algorithm for Frame Fragment Selection

Suppose the video sequence under study has total ' $n$ ' number of frames, then we will pass all the frames from frame number 1 to $n$ to frame fragment selection unit in way of current and next frame. If ' $i$ ' is the current frame then the unit will first estimate the Mean Square Error between ' $i$ ' and ' $i+1$ ' frame. Whenever system is given with RGB format video we will have more than two channels available in a frame. In such cases, the Mean Square Error (MSE) will be calculated for each channel of two successive frames under consideration and then average MSE of all channels will be considered as MSE value of two frames. Subsequently an array of MSE values of all the frames will be generated and from that array, selected frames involving major events can be selected.

$$
M S E=\frac{1}{M N} \sum_{n=1}^{M} \sum_{n=1}^{n}\left[i^{\wedge}(n, m)-i(n, m)\right]^{2}
$$

This novel frame selection unit drastically reduces the number of frames which are considered for feature extraction and as a result the computational cost is reduced.

\subsection{Feature Extraction}

Video feature are the basic elements to detect an activity from video. The outcome of the preprocessing stage is taken as a contribution to the feature extraction module. The goal of feature extraction is to find out basic discriminative data and representing it in an appropriate structure which can be given as a input to classifier stage. There is no all-inclusive applied list of features for any violent detection framework since nature of the video sequence from various monitoring territory by and large contain not many common characteristics. The errand of feature choice in the area of violent activity recognition is of prime significance since system effectiveness, memory necessity and processing time are mostly reliant on cardinality of set of features. An appropriate blend of features will be utilized to improve the classifier's efficiency to interestingly describe the circumstance in video. 


\subsubsection{Multi-Resolution Local Binary Pattern (LBP)}

Local Binary Pattern (LBP) is one of the most efficient texture operators which describes the pixels of an image by considering the neighborhood of each pixel, thresholding the pixels with respect to center pixel and converting the level in terms of binary number. LBP operator is prominently used in various applications because of its computational simplicity.

The local binary pattern (LBP) method proposed by [Ojala et al., (1996)] perform thresholding of gray level of neighbor pixels with the gray level of center pixel using equation

$$
L B P_{N R}=\sum_{n=0}^{N-1} f\left(i_{n}-i_{c}\right) 2^{n}
$$

The conventional LBP is inconvenient to use as a region descriptor because it generated long histograms. To overcome this issue [Liang et al., (2010)] computed LBP by comparing center symmetric pairs of pixels with the center pixel, rather than comparing each pixel with the center pixel using following equation which significantly decreases the total number of comparisons to be computed for neighbors.

Consider an arrangement of 8-neighbous as shown in the figure with a center pixel as Ic and 8-neighbous it I0 to I7.

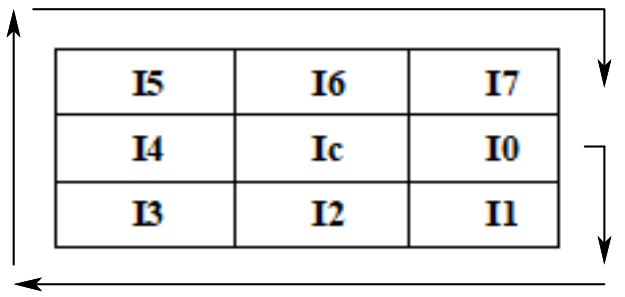

Fig 11: Example of modified local binary patterns for 8 neighboring pixels.

Firstly, thresholding of the center symmetric pairs of pixels with the center pixel using formulas as given in equation is done.

$$
\begin{aligned}
& D_{1}=\left(I_{0}>=I_{4}\right) \\
& D_{2}=\left(I_{1}>=I_{5}\right) \\
& D_{3}=\left(I_{2}>=I_{6}\right) \\
& D_{4}=\left(I_{3}>=I_{7}\right) \\
& D_{5}=\left(\frac{I_{0}+I_{1}+I_{2}+I_{3}+I_{4}+I_{5}+I_{6}+I_{7}}{8}\right)>=I_{C}
\end{aligned}
$$

Thus, the summative value of Modified LBP is calculated using equation

$$
M_{l b p}=2^{0} \times D_{1}+2^{1} \times D 2+2^{2} \times D 3+2^{3} \times D 4+2^{4} \times D 5
$$

The modified LBP generates only 32 (25) different binary patterns and can be used as Multi-Resolution LBP descriptor

\subsubsection{Fuzzy Histogram of Optical Flow Orientations}

In case of violence detection in video frame, one need to consider the features associated with motion sequence and estimation of optical flow is the best solution to motion related features. Optical flow when applied on consecutive frames, can bring out motion estimation of each pixel in its surrounding along both horizontal and vertical direction.

We estimate the optical flow for every frame with respect to previous frame in video sample using a inbuilt matlab function estimateFlow( ) which uses lucas kanade algorithm for optical flow estimation. Then computation of Fuzzy Histogram of Optical Flow Orientations is done using methods decribed in [S L Happy (2021)] by which we get appropriate angular histograms from optical flow vector orientations using histogram fuzzification. 


\subsection{Classifier}

Supervised learning algorithms are the class of machine learning, in which the model is developed by training the machines using well labelled training data, and then making use of this trained data, machines predict the output. The term labelled data refers to input data which is already tagged with the correct output data using some predefined set of rules.

In supervised machine learning techniques, the model trained on the basis training data works as advisor to machine for predicting the output correctly. This is analogous to the students learning in the supervision of teachers in a class.

In case of Supervised learning the input variables (A) and an output variable (B) can be correlate with following mapping function of input to the output.

$$
B=f(A)
$$

The objective is to estimate be best mapping function so that when we have a new input data say $(C)$, the algorithm must be able to forecast the output variable(B) for that new input data. The concept of Supervised learning technique can be elaborate with the help of following generalized block diagram.

Labeled Data

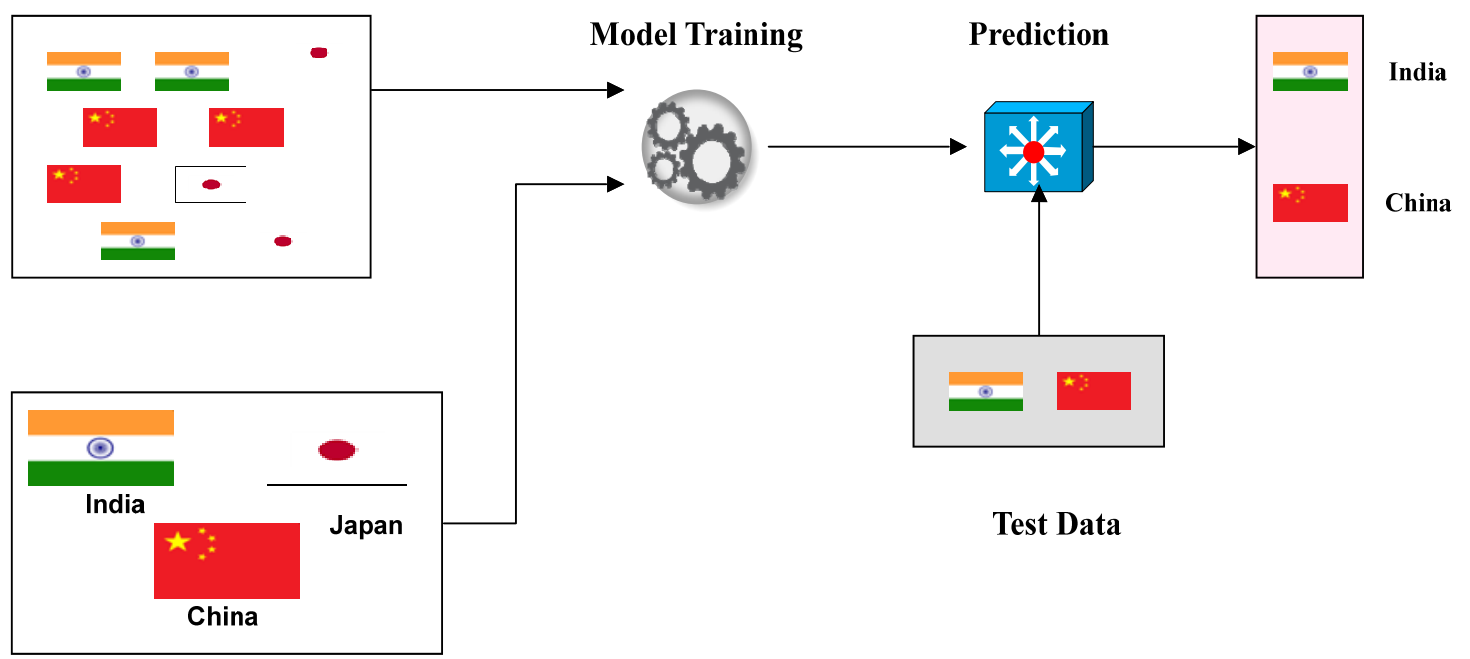

Labeles

Fig 12: Proposed Violent Video Classification System

Suppose we have a dataset of distinct types of flags of various countries which include flag of India, Japan and China and by means of a model we wanted to predict the unknow input flag image belongs to which country. Now the very first step here would be to train the model for each flag.

We will train the model with the help of labelled data using predefined set of rules as follows.

- If the flag image is of rectangular size and white banner with a crimson-red circle at its center, then it will be labelled as a national flag of Japan.

- If the flag image is of rectangular size with red background and five golden stars at left upper corner, then it will be labelled as a national flag of Japan.

- If the flag image is of rectangular size having three equally distributed colours of saffron, white and green with a 24-spoke wheel at center then it will be labelled as a national flag of India.

Now, once the training is over, we evaluate the model using the test dataset and the task of the model is to identify the input flag image belongs to which country.

The model which is already trained on all types of flag images, when given with input of new flag image, it classifies the input flag image on the basis of background colours, center shapes, stars and other details (as labelled during training) and predicts the correct output.

An ensemble machine learning algorithms falls in the category of supervised learning algorithms, since such category of algorithms can be trained and once trained, they can be used to make predictions on test samples. Ensemble boosting algorithm is a special class of algorithm which converts the weak learners into stronger learners by using weighted averages. Ensemble boosting algorithm works on building an ensemble by training every new model to improve the overall training keep in view of training instances that were mis-classified by previous models. In many cases, ensemble boosting algorithm outperforms the bagging algorithms on the scale of accuracy, but it may also cause model over-fitting over the training data. Most commonly used ensemble 
boosting algorithm is Adaboost in case of binary classification. Table 3 lists few of the prominent characteristics of the various ensemble algorithms.

\begin{tabular}{|c|c|c|c|c|c|}
\hline Algorithm & Regression & $\begin{array}{c}\text { Binary } \\
\text { Classification }\end{array}$ & $\begin{array}{c}\text { Multiclass } \\
\text { Classification }\end{array}$ & $\begin{array}{c}\text { Class } \\
\text { Imbalance }\end{array}$ & Stop \\
\hline Bag & $\checkmark$ & $\checkmark$ & $\checkmark$ & & \\
\hline Subspace & & $\checkmark$ & $\checkmark$ & & \\
\hline LPBoost & & $\checkmark$ & $\checkmark$ & & $\checkmark$ \\
\hline GentleBoost & & $\checkmark$ & & & \\
\hline RUSBoost & & $\checkmark$ & $\checkmark$ & $\checkmark$ & \\
\hline AdaBoostM1 & & $\checkmark$ & & & \\
\hline RobustBoost & & $\checkmark$ & & & $\checkmark$ \\
\hline TotalBoost & & $\checkmark$ & $\checkmark$ & & \\
\hline LogitBoost & & $\checkmark$ & & & \\
\hline AdaBoostM2 & & & $\checkmark$ & & \\
\hline LSBoost & $\checkmark$ & & & & \\
\hline
\end{tabular}

Table 1. Summary of prominent characteristics of the various ensemble algorithms

\section{Experimental Analysis}

In this section, the study of effect of two low level features and their fusion to the task of violent video detection and classification is reported. We evaluate the performance of proposed method and comparison of proposed violence detection method is done with the different state-of-the-are related methods reported in literature.

\subsection{Dataset}

Experimental analysis of proposed system is conducted on two benchmarking datasets as Hockey Fight Dataset and Peliculas Dataset which are commonly used in prior art. Both the dataset differs from each other in various characteristics such as variation in background, occlusion, motion and illumination conditions.

(1) Hockey Fight Dataset [Bermejo Nievas et al., (2011)]: This dataset contains fight (violent clips) and nonfight (non-violent) scenes taken from the footages of hockey games. In total there are 1000 video samples in the dataset, out of which 500 video samples belongs to violent sequences and rest 500 belongs to non-violent sequences. The length of videos is in the range of 1.5 to 2 seconds with a similar frame rate of 25 Frames / Second. Since, all the video belongs to set of ice hockey games, the background in all the videos appears to be similar.
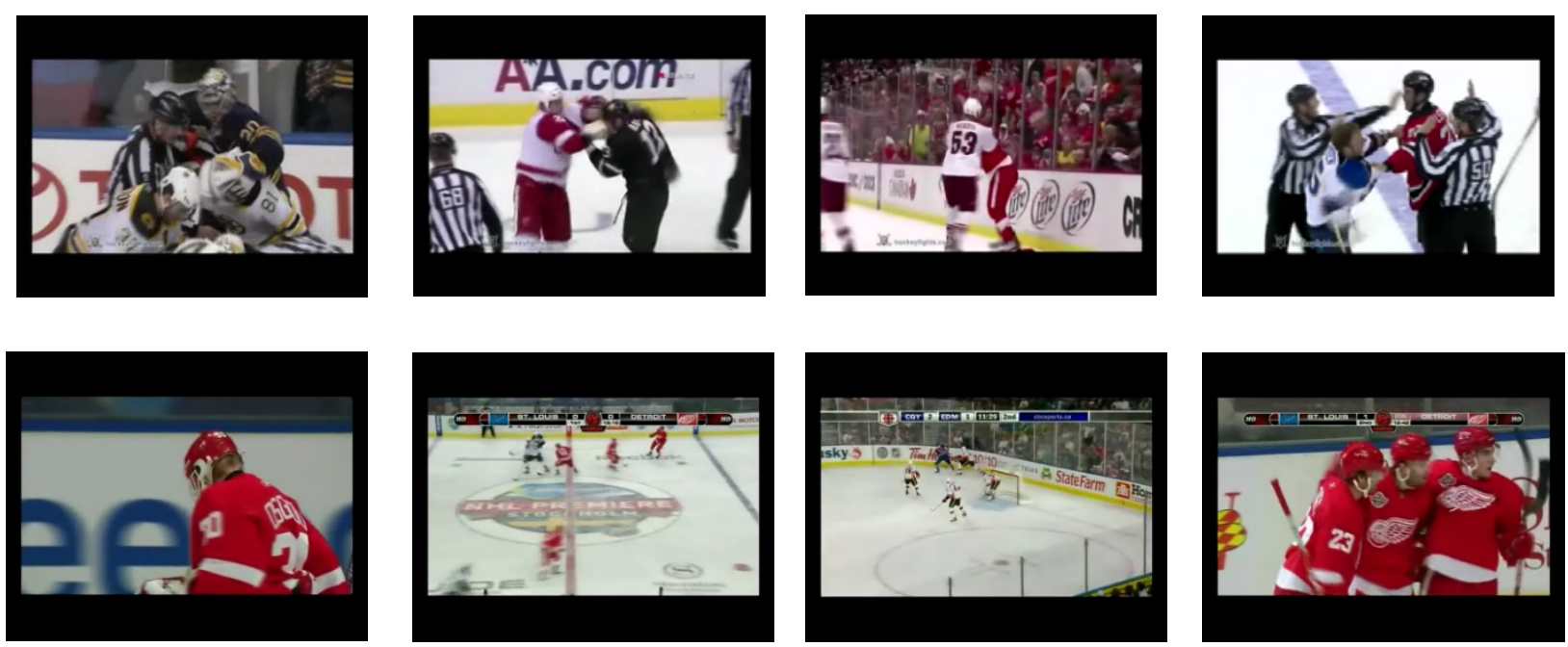

Fig 13: Sample frames from video sequences of violent class content (first row) and non-violent class content (second row) for Hockey datasets 

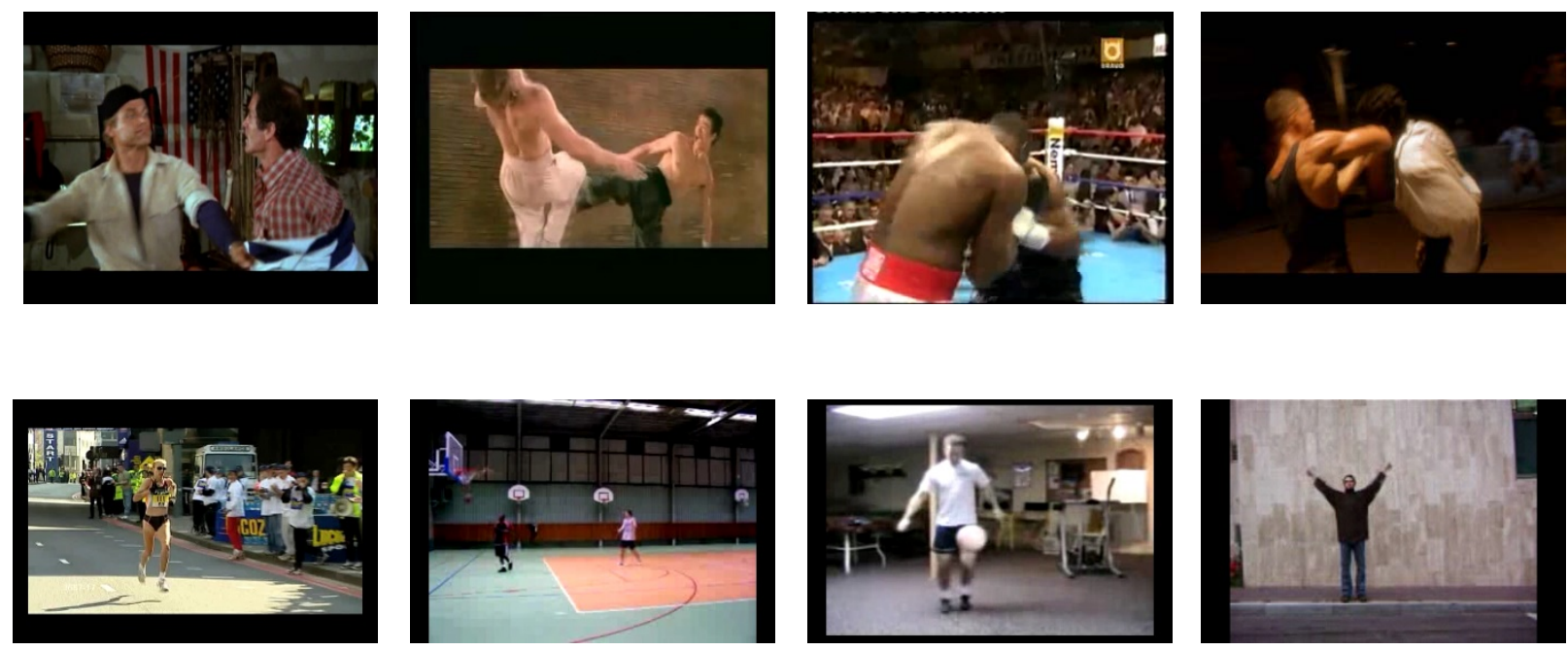

Fig 14: Sample frames from video sequences of violent class content (first row) and non-violent class content (second row) for Peliculas datasets

(2) Peliculas Dataset: This dataset contains fight (violent clips) and non-fight (non-violent) scenes taken from majorly Hollywood movies, football games and other major events. In total there are 200 video samples in the dataset, out of which 100 video samples belongs to violent sequences and rest 100 belongs to non-violent sequences. Here the background, environment and people in the video are varying, since the video clips are taken from different movie scenes. The maximum length of video clip is up to 2 seconds with a variable frame rate from 25 Frames / Second to 30 Frames / Seconds.

\begin{tabular}{|c|c|c|c|c|}
\hline Dataset & Category & Total No of Clips & Resolution & Duration (Seconds) \\
\hline Hockey Fight & Violent & $\mathbf{5 0 0}$ & $\mathbf{3 6 0} \times \mathbf{2 8 8}$ & \multirow{1}{*}{1 to 2 seconds long } \\
\cline { 2 - 4 } Dataset & Non-Violent & $\mathbf{5 0 0}$ & $\mathbf{3 6 0} \times \mathbf{2 8 8}$ & \\
\hline \multirow{2}{*}{ Peliculas Dataset } & Violent & $\mathbf{1 0 0}$ & Variable & \multirow{2}{*}{ to 2 seconds long } \\
\cline { 2 - 4 } & Non-Violent & $\mathbf{1 0 0}$ & Variable & \\
\hline
\end{tabular}

Table 2. Summary of dataset commonly used for violent action detection in prior art

\subsection{Performance Evaluation Parameters.}

In Machine Learning applications, performance measurement is a crucial task. Model evaluation metrics are required to assess model efficiency and performance. The Fig. 15 shows the concept of contingency matrix or often called as confusion matrix, which contains four sections as TP (true positive), FP (false positive), FN (false negative) and TN (true negative).

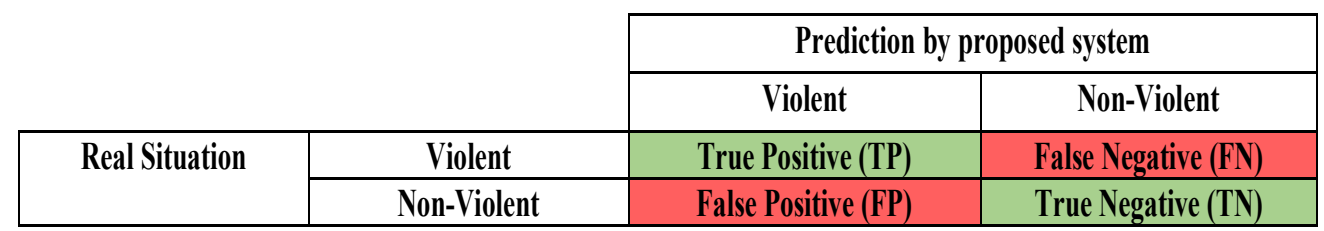

Fig 15: Generalized Confusion matrix for violence detection scenario

Suppose we have to major classes as violent (positive class) and non-violent (negative class). We can simply define TP, FP, TN and FN as follows.

- TP (True Positive) is the number of violent events (positive class) that the algorithm correctly predicted as violent.

- FP (False Positive) is the number of violent events (positive class) that the algorithm predicted incorrectly as non-violent.

- TN (True Negative) is the number of non-violent events (negative class) that the algorithm predicted correctly as non-violent. 
- FN (False Negative) is the number of non-violent events (negative class) that the model predicted incorrectly as violent.

Accuracy is a quality metric, which refers to how much near the actual value is the measured value; this metric us will help measure the accuracy of the inference of this model. By said Reason, in this context of identification the quality in terms of getting the highest TP and TN rate and simultaneously decrease FP and FN. Formally, accuracy has the following definition, as sample in (equation 3).

$$
\text { Accuracy }=\frac{\text { Number of correct Predictions }}{\text { Total Number of Predictions }}
$$

However, in binary classification, the accuracy can also be calculated in terms of positives and negatives, see (equation 4)

Where

$$
\text { Accuracy }=\frac{\mathrm{TP}+\mathrm{TN}}{\mathrm{TP}+\mathrm{TN}+\mathrm{FP}+\mathrm{FN}}
$$

- Accuracy: Total percentage of correct inferences.

- VP: Number of True Positive Results After of the model inference.

- VN: Number of True Negative Results after model inference.

- FP: Number of False Positive results after model inference.

- FN: Number of False Negative Results After the inference of the model.

The metric usually used in the context of binary classification is precision (or positive predictive value), which is defined in (equation 5), and measures the reliability of detection or proportion of TP cases among all positive cases detected by the test. Considering that TP + FP is the number of objects detected in each class, precision indicates what percentage of detections actually has value.

$$
\text { Precision }=\frac{\mathrm{TP}}{\mathrm{TP}+\mathrm{FP}}
$$

Where

- Precision: Percentage of correct inferences over the total scenes that the model inferred as violent.

- TP: Number of True Positive results after model inference.

- FP: Number of False Positive results after model inference.

As a third quality metric, the recall or completeness will be calculated. The recall measures the proportion of real positives that was correctly identified and is defined as follows (equation 6)

$$
\text { Recall }=\frac{\mathrm{TP}}{\mathrm{TP}+\mathrm{FN}}
$$

\section{Where}

- Recall: Percentage of correct inferences over the total of real cases (violence).

- TP: Number of True Positive results after model inference.

- FN: Number of False Negative results after model inference.

\subsection{Results.}

Experiments are performed on a computer with a Intel(R) Core-i3-5005U processor with a available maximum clock speed of $2.00 \mathrm{GHz}$, which has $4 \mathrm{~GB}$ of RAM without any Graphics Processing Unit (GPU) on windows 10 platform. The proposed technique is implemented with Matlab environment with statistical and machine learning toolbox and computer vision toolbox.

Table 2 reports the evaluated results with different feature conditions, when extracted frames from sample video is set to 5, with the classifier as Ensemble RobustBoost aggregation method for classification to two class specification having maximum number of decision splits per tree as 50. The trained model with such specifications is used for making predications on test data. Evaluation of trained model is done in terms of parameters such as computational time, accuracy, precision and recall for Hockey dataset and Peliculas dataset. 


\begin{tabular}{|c|c|c|c|c|c|c|c|c|}
\hline \multirow[b]{2}{*}{$\begin{array}{l}\text { Feature / } \\
\text { Features }\end{array}$} & \multicolumn{4}{|c|}{ Hockey Dataset } & \multicolumn{4}{|c|}{ Peliculas dataset } \\
\hline & $\begin{array}{l}\text { Computational } \\
\text { Time for } \\
\text { feature } \\
\text { extraction (in } \\
\text { seconds) }\end{array}$ & $\begin{array}{c}\text { Accuracy } \\
\text { (in \%) }\end{array}$ & $\begin{array}{l}\text { Precision } \\
\text { (in \%) }\end{array}$ & $\begin{array}{l}\text { Recall } \\
\text { (in \%) }\end{array}$ & $\begin{array}{l}\text { Computational } \\
\text { Time for } \\
\text { feature } \\
\text { extraction (in } \\
\text { seconds) }\end{array}$ & $\begin{array}{c}\text { Accuracy } \\
\text { (in \%) }\end{array}$ & $\begin{array}{l}\text { Precision } \\
\text { (in \%) }\end{array}$ & $\begin{array}{c}\text { Recall (in } \\
\% \text { ) }\end{array}$ \\
\hline $\begin{array}{l}\text { Fuzzy Histogram } \\
\text { of Optical Flow } \\
\text { Orientations } \\
\text { (FHOH) }\end{array}$ & 1033.52 & 73.66 & 72.04 & 77.33 & 540.17 & 75 & 74.19 & 76.66 \\
\hline $\begin{array}{l}\text { Multi-Resolution } \\
\text { Local Binary } \\
\text { Patterns (MLBP) }\end{array}$ & 617.28 & 90.33 & 86.66 & 95.33 & 283.67 & 91.66 & 90.32 & 93.33 \\
\hline $\begin{array}{l}\text { Fuzzy Histogram } \\
\text { of Optical Flow } \\
\text { Orientations + } \\
\text { Multi-Resolution } \\
\text { Local Binary } \\
\text { Patterns }\end{array}$ & 876.36 & 88.66 & 85.80 & 92.66 & 531.96 & 91.7 & 93.1 & 90 \\
\hline
\end{tabular}

Table 3. Result Analysis of proposed algorithm with respect to Accuracy, Precision and Recall

The computation time required to extract the various features is of primary importance when detecting the violent action in real time such as in case of visual surveillance. Fig. 1 shows the graphical representation of computational time required in seconds to extract Fuzzy Histogram of Optical Flow Orientations (FHOH), Multi-Resolution Local Binary Patterns (MLBP) and combination of Fuzzy Histogram of Optical Flow Orientations \& Multi-Resolution Local Binary Patterns for Hockey Fight Dataset and Peliculas Dataset.

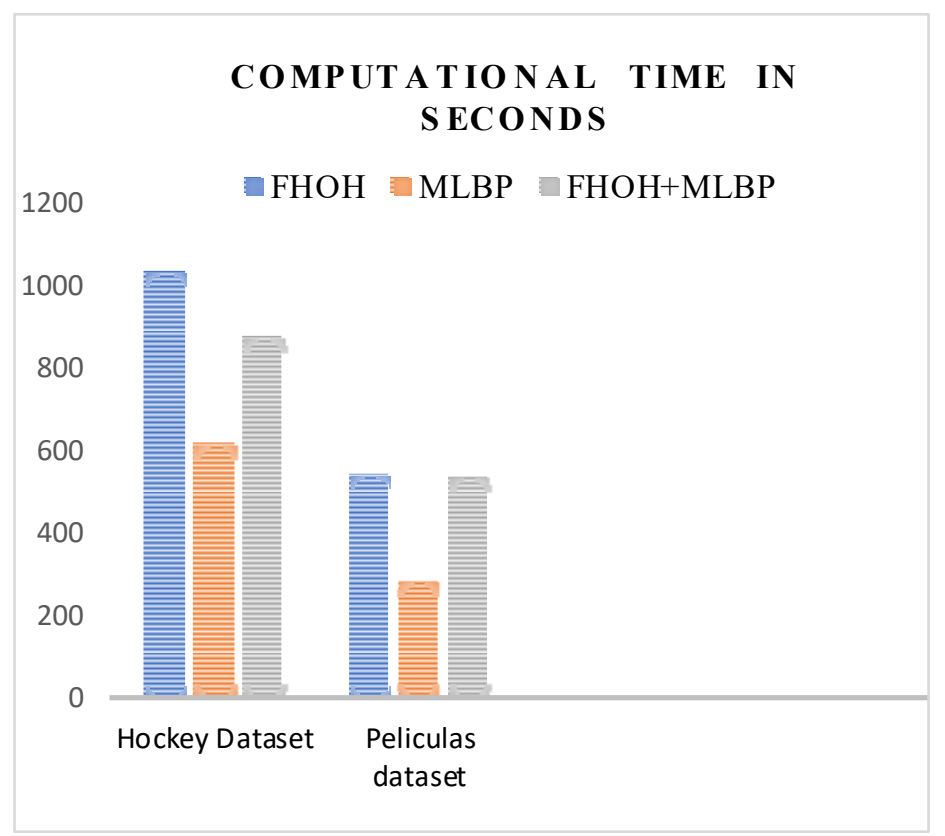

Fig 16: Computational time in seconds for different feature(s) extraction on Hockey Fight Dataset and Peliculas Dataset

Multi-Resolution Local Binary Patterns feature takes minimum time and Fuzzy Histogram of Optical Flow Orientations takes maximum time for extraction on both the datasets. ROC curve (receiver operating characteristic curve) for violence prediction and confusion matrices for various combinations of features on both the datasets are shown below from Fig. 1 to Fig. 8 . 


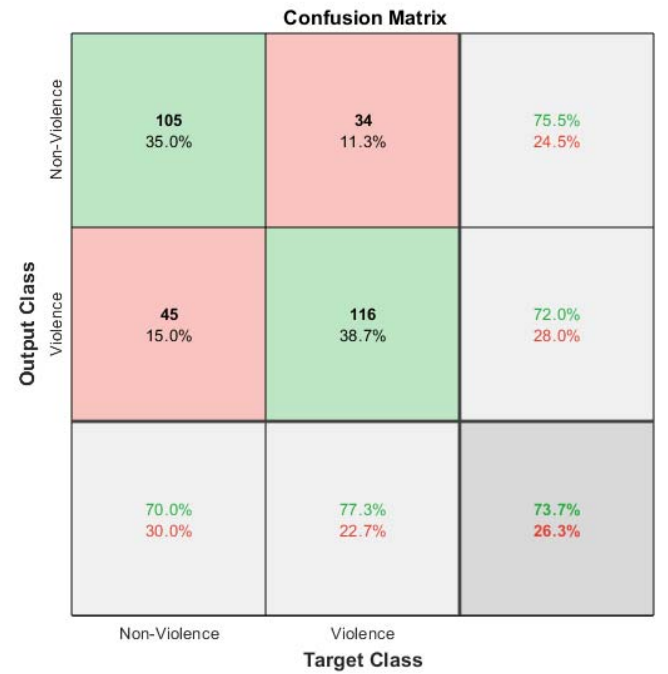

(a)

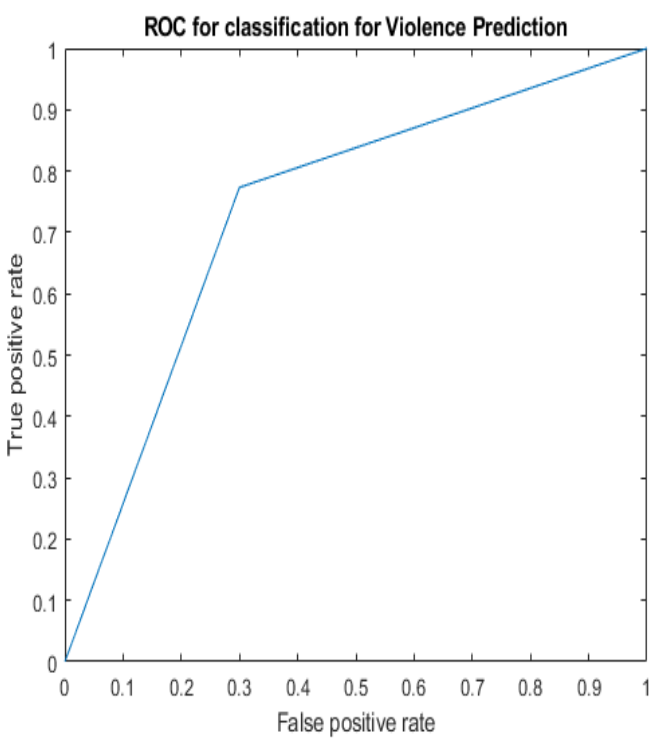

(b)

Fig 17: (a) Confusion matrix and (b) ROC curve considering Fuzzy Histogram of Optical Flow Orientations (FHOH) feature for Hockey Fight Dataset

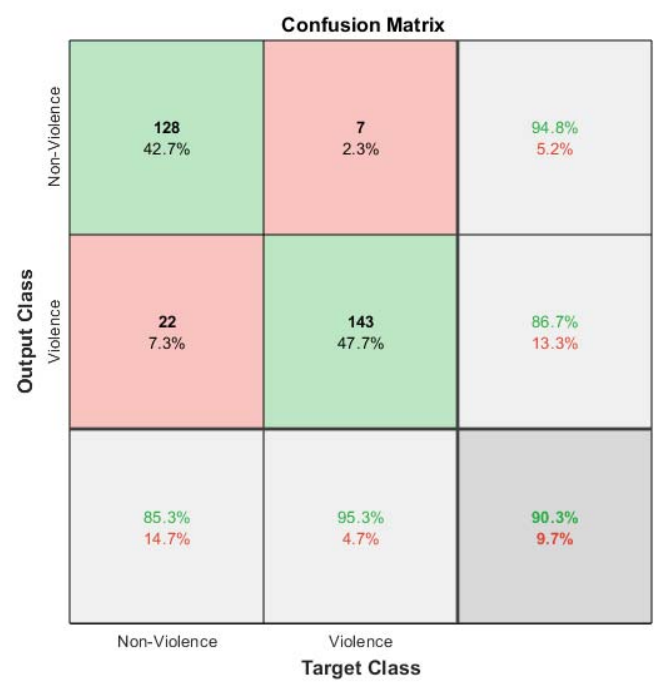

(a)

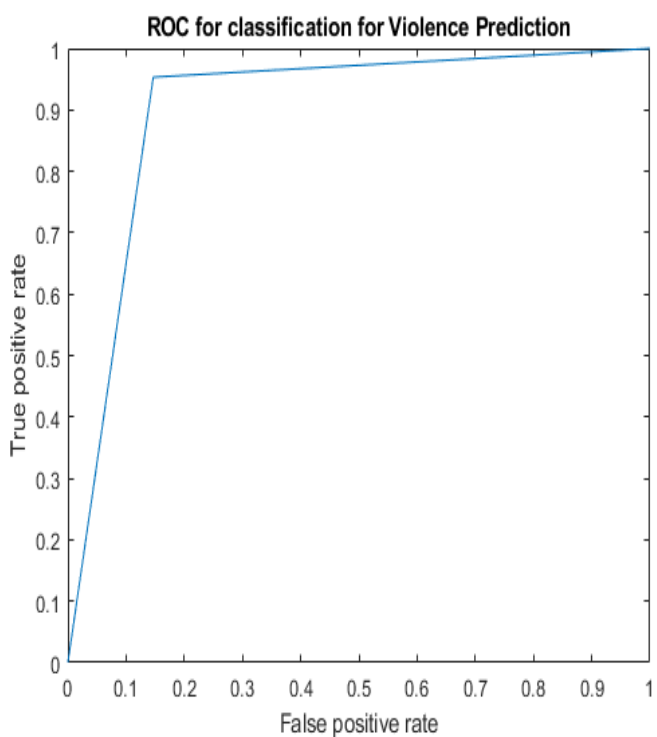

(b)

Fig 18: (a) Confusion matrix and (b) ROC curve considering Multi-Resolution Local Binary Patterns (MLBP) feature for Hockey Fight Dataset 


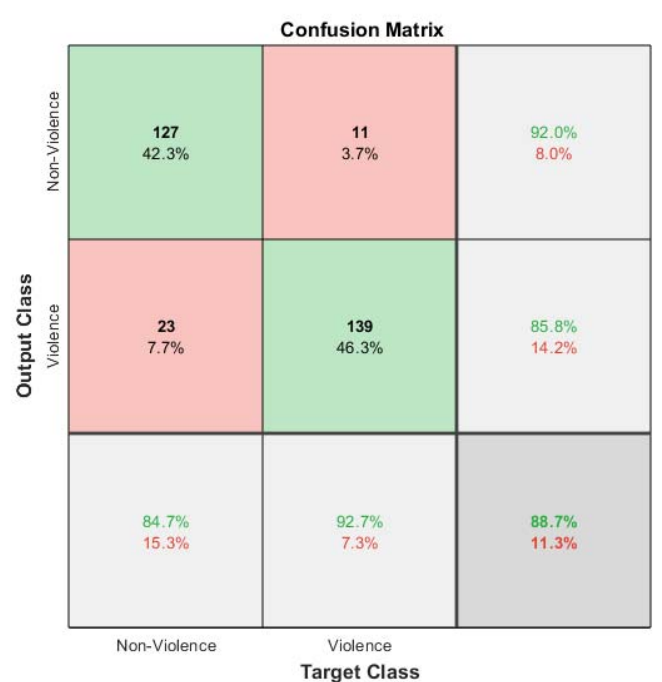

(a)

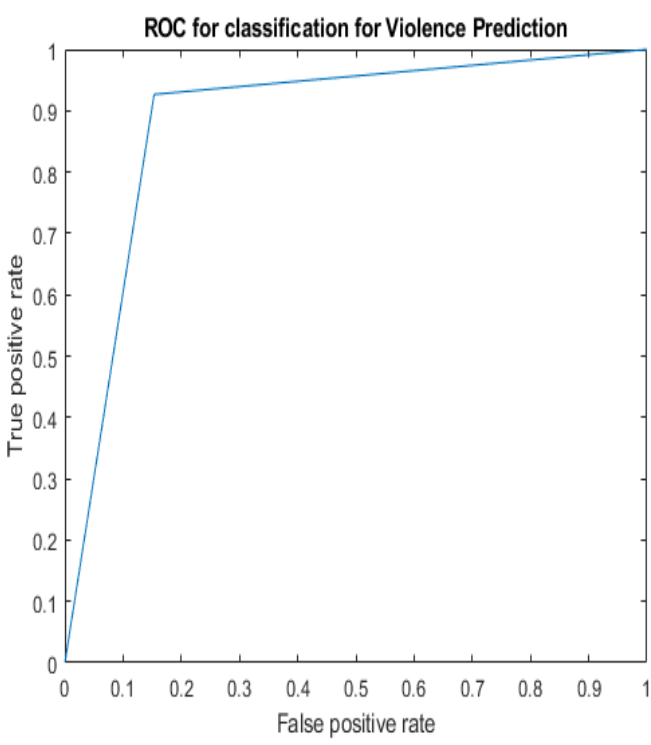

(b)

Fig 19: (a) Confusion matrix and (b) ROC curve considering Fuzzy Histogram of Optical Flow Orientations and Multi-Resolution Local Binary Patterns feature for Hockey Fight Dataset

From the confusion matrices and roc curves it is evident that the Multi-Resolution Local Binary Patterns (MLBP) seems to be more suitable for classification of violent category of video in case of Hockey Fight Dataset. Studeies also shows that Fuzzy Histogram of Optical Flow Orientations (FHOFH) alone can not give significant results for accurate classification in Hockey Fight Dataset and Peliculas Dataset.

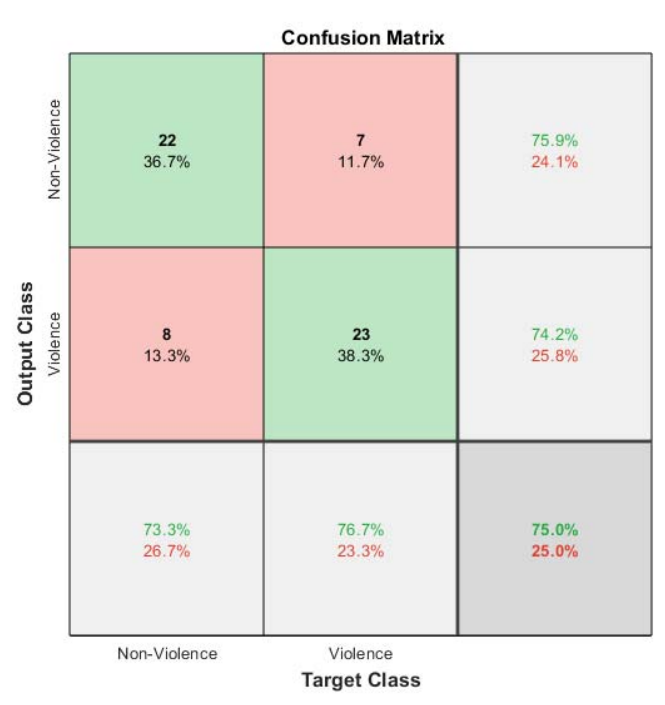

(a)

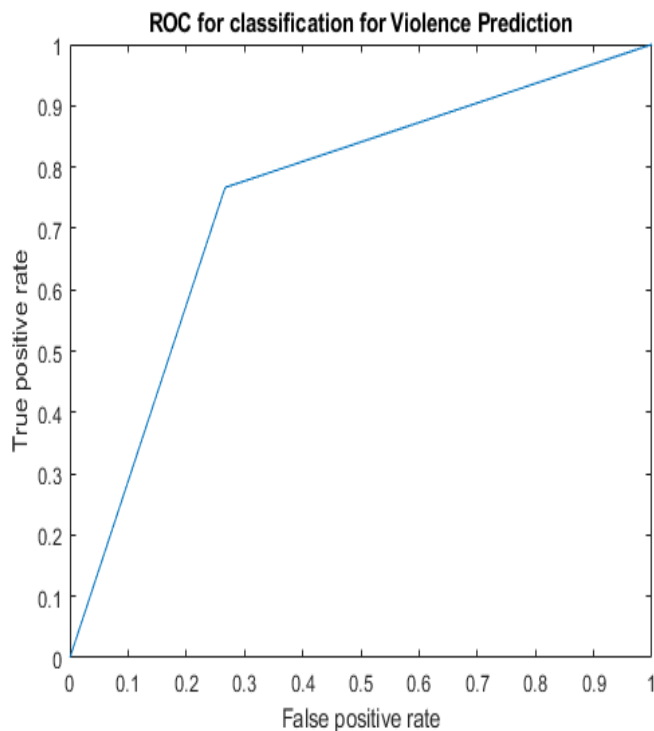

(b)

Fig 20: (a) Confusion matrix and (b) ROC curve considering Fuzzy Histogram of Optical Flow Orientations (FHOH) feature for Peliculas Dataset 


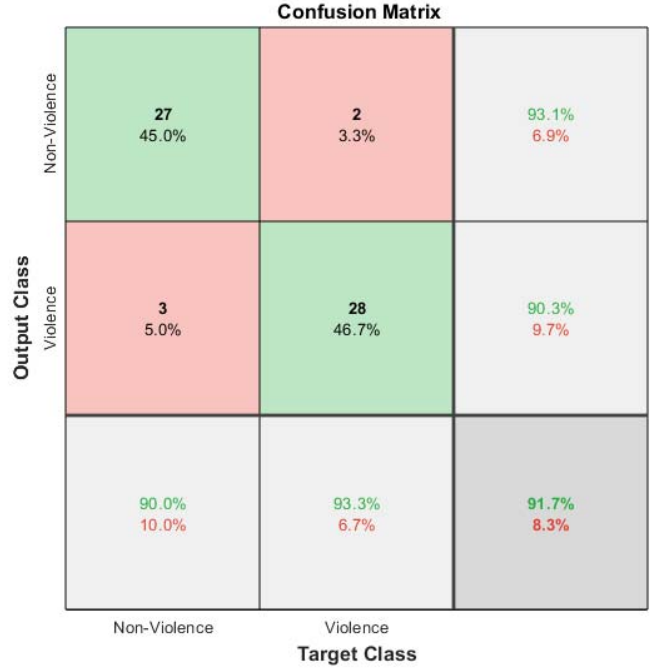

(a)

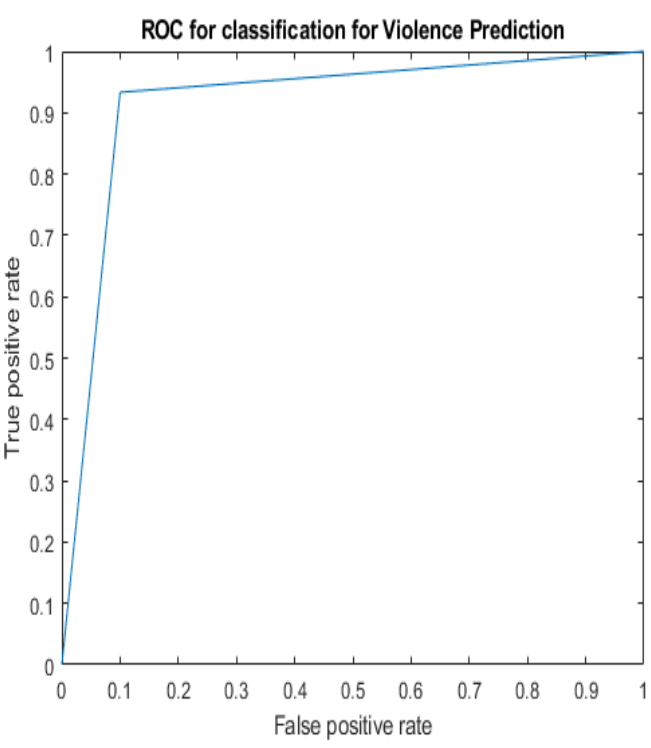

(b)

Fig 21: (a) Confusion matrix and (b) ROC curve considering Multi-Resolution Local Binary Patterns (MLBP) feature for Peliculas Dataset

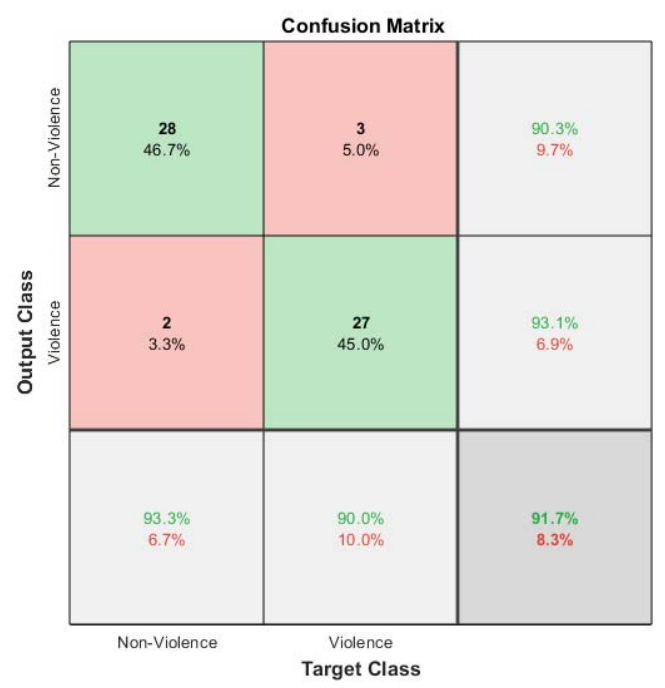

(a)

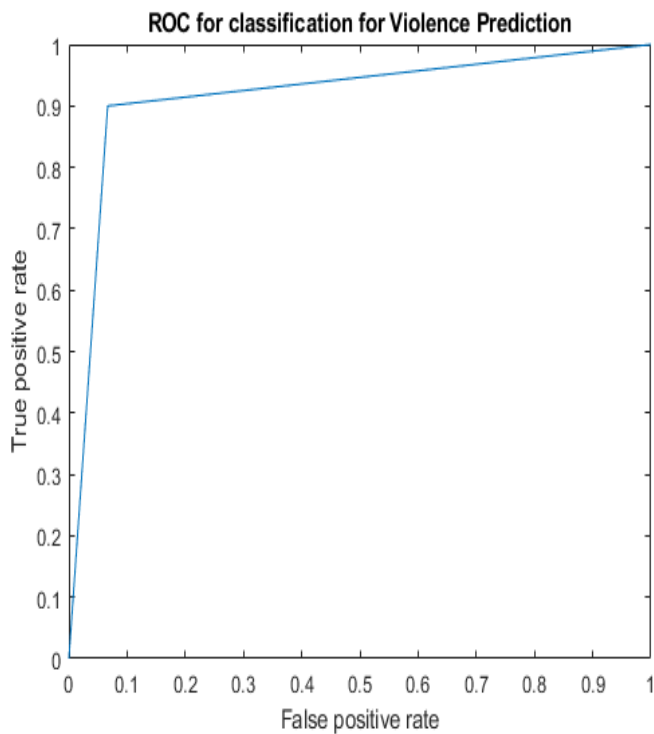

(b)

Fig 22: (a) Confusion matrix and (b) ROC curve considering Fuzzy Histogram of Optical Flow Orientations and Multi-Resolution Local Binary Patterns feature for Peliculas Dataset

In addition, we evaluate the performance proposed algorithms with similar methods reported in literature by selecting the configuration with the optimal accuracy values. Table 5 describes the comparative analysis of proposed violence detection method on Hockey dataset and Peliculas dataset with the prominent methods reported in literature. The experimental results are close to state-of-the-art. 


\begin{tabular}{|l|c|}
\hline \multicolumn{1}{|c|}{ Method and Classifier } & $\begin{array}{c}\text { Accuracy } \\
\mathbf{( \% )}\end{array}$ \\
\hline (Random Forest) [Das et al., (2019)] & 86 \\
\hline (Random Forest) [Gracia et al., (2015)] & 82.4 \\
\hline (Adaboost) [Gracia et al., (2015)] & 71.7 \\
\hline 2D-CNN [Serrano et al., (2018)] & 87.8 \\
\hline MoSIFT +BoW [Zhang et al., (2017)] & 88.8 \\
\hline MoWLD+ BoW [Zhang et al., (2017)] & 89.28 \\
\hline Random Forest [Lloyd et al., (2016)] & 84.6 \\
\hline Linear SVM [Lloyd et al., (2016)] & 87.4 \\
\hline ResNet50 + LSTM (SoftmaX) [Ditsanthia et al., (2019)] & 83.19 \\
\hline VGG19 + LSTM (SoftmaX) [Ditsanthia et al., (2019)] & 80.16 \\
\hline Xception + LSTM (SoftmaX) [Ditsanthia et al., (2019)] & 79.04 \\
\hline Proposed Method & $\mathbf{9 1}$ \\
\hline
\end{tabular}

Table 4. Comparison of proposed violence detection algorithm performance on hockey fight dataset and Peliculas dataset with state-ofthe-art methods

\section{Conclusion}

The proposed framework has been extensively tested on two challenging datasets involving with public violence, person-on-person violence and real-world violence videos captured from cctv. The proposed method shows good accuracy and improves upon multiple algorithms reported in literature. And it potentially contributes in improving the computational time requirement of methods of violence activity detection from surveillance videos with maintaining the reasonable accuracy.

Future work will be in the direction to compare the proposed approach with other deep learning methods specifically extracting spatio-temporal details in video and estimating the sustainability of present method for more generic action recognition task for other areas in computer vision.

\section{Acknowledgments}

The authors are thankful to G H Raisoni University, Amravati and the department of Applied Electronics, Sant Gadge Baba Amravati University for providing research facilities.

\section{References}

[1] Cristani, M., Bicego, M., Murino, V., 2007. Audio-Visual Event Recognition in Surveillance Video Sequences. IEEE Trans. Multimed. 9, 257-266. https://doi.org/10.1109/TMM.2006.886263

[2] Das, S., Sarker, A., Mahmud, T., 2019. Violence Detection from Videos using HOG Features. 2019 4th Int. Conf. Electr. Inf. Commun. Technol. EICT 2019 20-22. https://doi.org/10.1109/EICT48899.2019.9068754

[3] Ditsanthia, E., Pipanmaekaporn, L., Kamonsantiroj, S., 2019. Video Representation Learning for CCTV-Based Violence Detection. TIMES-iCON 2018 - 3rd Technol. Innov. Manag. Eng. Sci. Int. Conf. 1-5. https://doi.org/10.1109/TIMES-iCON.2018.8621751

[4] Gracia, I.S., Suarez, O.D., Garcia, G.B., Kim, T.K., 2015. Fast fight detection. PLoS One 10, 1-19. https://doi.org/10.1371/journal.pone.0120448

[5] Hassner, T., Itcher, Y., Kliper-Gross, O., 2012. Violent flows: Real-time detection of violent crowd behavior. IEEE Comput. Soc. Conf. Comput. Vis. Pattern Recognit. Work. 1-6. https://doi.org/10.1109/CVPRW.2012.6239348

[6] Jaiswal, S.G., Mohod, S.W., 2021. Implementation of violence detection system using soft computing approach. Lect. Notes Data Eng. Commun. Technol. 54, 737-748. https://doi.org/10.1007/978-981-15-8335-3_56

[7] Jaiswal, S.G., Mohod, S.W., 2020. Recapitulating the Violence Detection Systems. Lect. Notes Electr. Eng. 570, $197-203$. https://doi.org/10.1007/978-981-13-8715-9_25

[8] Keçeli, A.S., Kaya, A., 2017. Violent activity detection with transfer learning method. Electron. Lett. 53, $1047-1048$. https://doi.org/10.1049/el.2017.0970

[9] Khokher, M.R., Bouzerdoum, A., Phung, S.L., 2015. Violent Scene Detection Using a Super Descriptor Tensor Decomposition. 2015 Int. Conf. Digit. Image Comput. Tech. Appl. DICTA 2015. https://doi.org/10.1109/DICTA.2015.7371320

[10] Liang, P., Li, S.F., Qin, J.W., 2010. Multi-resolution local binary patterns for image classification. 2010 Int. Conf. Wavelet Anal. Pattern Recognition, ICWAPR 2010 164-169. https://doi.org/10.1109/ICWAPR.2010.5576318

[11] Lloyd, K., Rosin, P.L., Marshall, A.D., Moore, S.C., 2016. Violent behaviour detection using local trajectory response. IET Semin. Dig. 2016. https://doi.org/10.1049/ic.2016.0082

[12] Ojala, T., Pietikäinen, M., Harwood, D., 1996. A comparative study of texture measures with classification based on feature distributions. Pattern Recognit. 29, 51-59. https://doi.org/10.1016/0031-3203(95)00067-4

[13] M. Hörhan and H. Eidenberger, "New content-based features for the distinction of violent videos and martial arts," 2013 IEEE International Conference on Acoustics, Speech and Signal Processing, 2013, pp. 1836-1839, doi: 10.1109/ICASSP.2013.6637970.

[14] Ramzan, M., Abid, A., Khan, H.U., Awan, S.M., Ismail, A., Ahmed, M., Ilyas, M., Mahmood, A., 2019. Violence Detection Techniques. IEEE Access 7, 107560-107575. 
[15] Senst, T., Eiselein, V., Kuhn, A., Sikora, T., 2017. Crowd Violence Detection Using Global Motion-Compensated Lagrangian Features and Scale-Sensitive Video-Level Representation. IEEE Trans. Inf. Forensics Secur. 12, 2945-2956. https://doi.org/10.1109/TIFS.2017.2725820

[16] Senst, T., Eiselein, V., Sikora, T., 2015. A local feature based on lagrangian measures for violent video classification. IET Semin. Dig. 2015. https://doi.org/10.1049/ic.2015.0104

[17] Serrano, I., Deniz, O., Espinosa-Aranda, J.L., Bueno, G., 2018. Fight Recognition in Video Using Hough Forests and 2D Convolutional Neural Network. IEEE Trans. Image Process. 27, 4787-4797. https://doi.org/10.1109/TIP.2018.2845742

[18] Souza, F. De, Pedrini, H., 2017. Detection of Violent Events in Video Sequences Based on Census Transform Histogram. Proc. - 30th Conf. Graph. Patterns Images, SIBGRAPI 2017 323-329. https://doi.org/10.1109/SIBGRAPI.2017.49

[19] Sudhakaran, S., Lanz, O., 2017. Learning to detect violent videos using convolutional long short-term memory. 2017 14th IEEE Int. Conf. Adv. Video Signal Based Surveillance, AVSS 2017. https://doi.org/10.1109/AVSS.2017.8078468

[20] Tang, Y.P., Wang, X.J., Lu, H.F., 2009. Intelligent video analysis technology for elevator cage abnormality detection in computer vision. ICCIT 2009 - 4th Int. Conf. Comput. Sci. Converg. Inf. Technol. 1252-1258. https://doi.org/10.1109/ICCIT.2009.206

[21] Wang, Y., Kato, J., 2016. Video-level violence rating with rank prediction. Proc. - 3rd IAPR Asian Conf. Pattern Recognition, ACPR 2015 071-075. https://doi.org/10.1109/ACPR.2015.7486468

[22] Xu, L., Gong, C., Yang, J., Wu, Q., Yao, L., 2014. VIOLENT VIDEO DETECTION BASED ON MoSIFT FEATURE AND SPARSE CODING Institution of Image Processing and Pattern Recognition, Shanghai Jiao Tong University, China School of Computing and Communications, University of Technology, Sydney, Australia. 2014 IEEE Int. Conf. Acoust. Speech Signal Process. 3562-3566.

[23] Zhang, T., Jia, W., He, X., Yang, J., 2017. Discriminative Dictionary Learning with Motion Weber Local Descriptor for Violence Detection. IEEE Trans. Circuits Syst. Video Technol. 27, 696-709. https://doi.org/10.1109/TCSVT.2016.2589858

[24] Zin, T.T., Kurohane, J., 2016. Visual analysis framework for two-person interaction. 2015 IEEE 4th Glob. Conf. Consum. Electron. GCCE 2015 519-520. https://doi.org/10.1109/GCCE.2015.7398694

[25] S L Happy (2021). Fuzzy Histogram of (FHOFO)(https://www.mathworks.com/matlabcentral/fileexchange/64804-fuzzy-histogram-of-optical-flow-orientations-fhofo), MATLAB Central File Exchange. Retrieved December 14, 2021.

[26] Serrano, I., Deniz, O., Espinosa-Aranda, J.L., Bueno, G., 2018. Fight Recognition in Video Using Hough Forests and 2D Convolutional Neural Network. IEEE Trans. Image Process. 27, 4787-4797. https://doi.org/10.1109/TIP.2018.2845742

[27] Bermejo Nievas E., Deniz Suarez O., Bueno García G., Sukthankar R. (2011) Violence Detection in Video Using Computer Vision Techniques. In: Real P., Diaz-Pernil D., Molina-Abril H., Berciano A., Kropatsch W. (eds) Computer Analysis of Images and Patterns.CAIP 2011. Lecture Notes in Computer Science, vol 6855. Springer, Berlin, Heidelberg. https://doi.org/10.1007/978-3-64223678-5 39

\section{Authors Profile}

\begin{tabular}{|l|l|}
\hline Snehil G Jaiswal - received B.E. degree in Electronics \& Telecommunication \\
Engineering and M.E. in Digital Electronics from Sant Gadge Baba Amravati \\
University in 2010 and 2014. He is presently pursuing Ph.D. from Sant Gadge Baba \\
Amravati University. In 2010, he joined G H Raisoni College of Engineering \& \\
Management Amravati, and presently working as officiating Registrar at G H Raisoni \\
University, Amravati. His research areas include Image Processing, Video Processing, \\
Action Recognition, Computer Vision and Machine learning.
\end{tabular}

